\title{
Memoria fragmentada: la reconstrucción del pasado en el ciberespacio
}

\author{
Fragmented memory: the reconstruction of the past \\ in cyberspace
}

(D) Andrea Angulo

andreateresa.angulo01@estudiant.upf.edu

Universidad Pompeu Fabra (Barcelona, España)

https://orcid.org/0000-0001-7275-9778

DOI https://doi.org/10.32719/26312514.2020.3.4

\section{Resumen}

En la era digital, la relación de los sujetos con el pasado ha cambiado por el sentido de inmediatez de internet y la expansión de la memoria de los dispositivos electrónicos. El presente es el marco temporal dominante, debido a la actualización permanente de información al que se tiene acceso cotidianamente desde las tecnologías comunicacionales. En el presente ensayo, se analizará el proceso de transformación del sentido del pasado y las nuevas estrategias para construirlo. Para ello, se realizará una discusión entre la comunicación digital y la historiografía que permitirá determinar la influencia del uso de internet en las maneras de crear memoria actualmente y la incidencia de este fenómeno en el trabajo de los historiadores.

\section{Abstract}

In the digital era, the relationship of the subjects with the past has changed due to the sense of immediacy of the internet and the expansion of the memory of electronic devices. The present is the dominant time frame, due to the permanent updating of information that is accessed daily from communication technologies. In this essay, the process of transforming the sense of the past and the new strategies to build it will be analyzed. For this, there will be a discussion between Digital Communication and Historiography that will determine the influence of the use of the internet in the ways of creating memory today and the incidence of this phenomenon in the work of historians.

\section{Palabras clave $\cdot$ Keywords}

Memoria; Internet; cultura digital; historiografía.

Memory; internet; digital culture; historiography. 


\section{Introducción}

El recuerdo implica un reencuentro con el pasado, pero ¿qué sucede cuando ese proceso está mediado por las tecnologías de la información? En la actualidad, la memoria humana se ha situado hacia lo digital, por medio del uso de aplicaciones que gestionan los contenidos en los diferentes dispositivos tecnológicos. Las prácticas tradicionales de conservación de vivencias han quedado para generaciones pasadas, ya que todos los acontecimientos se guardan en la nube y se reproducen en el presente en plataformas de medios sociales.

La actualización permanente de contenidos y la globalización cultural median el proceso de construcción de la memoria colectiva y la formación de las identidades. Los grandes relatos históricos se enfrentan a las narraciones particulares de los usuarios que muestran versiones alternas a las institucionalizadas desde el poder. Ahora, el concepto de Historia, (con mayúscula) está fragmentado por la aparición de voces diversas que se expresan mediante narraciones transmedia y se difunden en blogs, podcast, videos, álbumes digitales, entre otras plataformas.

En la cotidianidad y en los espacios académicos, como programas de posgrado, se está posicionando el debate sobre la dependencia, cada vez más notoria, de las tecnologías comunicacionales en las actividades humanas. Con respecto a la memoria, se ha hecho costumbre utilizar al teléfono inteligente como un asistente personal que recuerda por los usuarios desde reuniones de trabajo hasta cumpleaños de familiares. Igualmente, sirve como diario de notas y agenda telefónica.

Con estos antecedentes, el presente trabajo analizará cómo se crea y se desarrolla la memoria dentro de la era digital, mediante la discusión entre la Comunicación y la disciplina histórica. Para esto, se revisará la configuración de la percepción del tiempo en la actualidad, las estrategias del poder para construir un sentido del pasado. Igualmente, se abordará la creación del relato sobre la memoria dentro de internet, la presencia de la ficción como nuevo medio para construcción de identidades y mitos, y el uso de internet como repositorio de conocimiento y fuente para la operación historiográfica.

\section{2. ¿Cómo se piensa sobre el pasado? Una aproximación a la concepción del tiempo}

Según Laura Moya y Margarita Olvera (2010, 448), las sociedades actuales han fracturado su percepción del tiempo, ya que cuestionan los supuestos y los marcos de interpretación, mediante los que las generaciones anteriores se ubicaban en la realidad. Esta ruptura se origina en la crisis que enfrenta la Modernidad, como proyecto histórico; sus planes comenzaron a desmoronarse con el fin de la Guerra Fría y el terrorismo de inicios del siglo XXI (447). 
Estas transformaciones han dirigido las discusiones teóricas acerca de la definición de este nuevo horizonte temporal, al cual se ha nombrado como Tardomodernidad. De acuerdo con Moya y Olvera, esta etapa se caracteriza por estos factores: la expansión global de interdependencias, la ausencia de un centro político, la desterritorialización del capitalismo, la migración masiva, la erosión de categorías sociales, la diversificación de los marcos de sentido, la individualización, los acelerados cambios tecnológicos y la compactación de espacio y tiempo, en relación con la percepción contraída del futuro (448).

Sobre estos puntos, se debe considerar que el crecimiento de la individualidad y el rápido desarrollo de la tecnología son los parámetros que más se están acentuando dentro de la sociedad de la información, a la que se refiere Castells (2009). La presencia ubicua de las tecnologías comunicacionales incide en el consumo individualizado de contenidos, lo cual ha fraccionado el concepto de verdad absoluta y, por tanto, de los grandes relatos.

Para Zygmunt Bauman (2002), la Modernidad ha provocado la ruptura de la tradición mediante un discurso que apuesta por el progreso. Según el sociólogo, esto implica convertir en estado líquido las estructuras establecidas para permitir la fluidez del cambio. Por esto, señala que los ideales modernos son los que han causado el desapego de los sujetos con el pasado (9).

El actual fracaso de la Modernidad como proyecto ideológico ha modificado la percepción del presente y del futuro. En el siglo anterior, eran vistos con optimismo. Sin embargo, ahora, el porvenir es motivo de incertidumbre, lo cual ha ubicado al presente como el marco temporal dominante. Según Moya y Olivera (2002, 451), "pareciera ser un tiempo que se basta a sí mismo", es decir, que el presente no reconoce al pasado como herencia, ni se proyecta hacia el futuro, sino que actúa a través de una pulsión a lo inmediato, lo nuevo y lo fugaz (451). Dentro de ese fenómeno, las NTIC tienen un papel protagónico, pues mantienen a los usuarios en un estado de actualidad permanente.

\section{Memoria y recuerdo: reconstrucción del pasado}

Si el pasado ha perdido relevancia, cabe preguntarse ¿cómo construyen la memoria las sociedades actuales? Para Maurice Halbwachs (1968, 210-1), el recuerdo es un tejido social vivo, que se compone de las representaciones del pasado creadas y transmitidas por grupos de pertenencia, las cuales se modifican o renuevan con el paso del tiempo. Halbwachs definió a este constructo como memoria colectiva.

Desde el punto de vista historiográfico, este concepto deja por fuera la experiencia individual, al asumir que la naturaleza social del pensamiento es un factor determinante en la configuración del ser entendido recuerdo (Moya y Olvera 2010, 439). Sin embargo, para los fines de este trabajo, se ocupará esta definición para comprender cómo se creaba el recuerdo colectivo antes de la era de internet. 
Como punto de partida, se puede hablar de la creación de la memoria de la nación. Esta es una de las construcciones sociales más relevantes para una comunidad, dado que corresponde a la narración oficial de un país; su finalidad es consolidar un sentido de identidad nacional y de pertenencia a un territorio. Para su formación es preciso negociar sobre lo que se va a recordar, lo que se olvidará y de qué manera se acudirá al pasado (Bustos 2007, 112).

Este proceso es llevado a cabo a partir de políticas de memoria establecidas por el poder estatal. Según Elizabeth Jelin $(2002,49)$, estas herramientas son accionadas por "emprendedores de la memoria", que son actores sociales que buscan legitimar una versión del pasado por medio de acciones de visibilización. En Latinoamérica los intelectuales cumplieron este papel, ya que adaptaron el pasado, según los intereses de las élites sociales y políticas (41).

Jelin explica que la memoria nacional se caracteriza por transmitir una versión "ejemplar" del pasado, es decir, un relato que permita educar a la población para aprender principios y valores que modelen las acciones en el presente (58). Por ello, se reconstruyen los hechos desde el lugar de enunciación de los vencedores, a partir del cual se seleccionan héroes, villanos y escenarios. Esta estrategia implica desplazar a los relatos marginales (que habitaban en la oralidad o en prácticas de resistencia), porque proporcionaban "sentidos diferentes del pasado, amenazando el consenso nacional que se pretende imponer" (41).

Para cualquier tipo de poder, es necesario visibilizar su perspectiva de la realidad (en este caso, del pasado), ya que de esta manera normaliza su discurso. Con ese fin, instrumentaliza la educación tradicional y a los medios de comunicación, ya que son los principales canales de transmisión de la ideología. La difusión y reproducción masivas de las narrativas oficiales, mediante programas académicos y libros de texto, y en los diferentes productos mediáticos inciden en el imaginario colectivo, porque realizan un proceso de mediación sobre la base de lo que el Estado y los grupos hegemónicos han decido recordar y olvidar. Así, mientras más se difunda su versión de los hechos más control tiene del pensamiento colectivo.

Según José María Jardim (2010, 38), hoy, las sociedades se manejan bajo el principio de la economía de la información, lo cual significa reconocer que los datos funcionan como el nuevo capital. El Estado moderno lleva ventaja, ya que es una de las fuentes más relevantes de información, debido a que tiene el control de archivos, bibliotecas, centros de documentación, entre otros (39). Sin embargo, con la llegada de internet, las personas pueden acceder libre y fácilmente a los contenidos y difundir sus propias visiones del pasado en sitios web.

Es un hecho que la popularización del uso de internet ha cambiado las relaciones de poder. Según Manuel Castells, la globalización multinivel — que se desprende del proce- 
so de digitalización - ha desestabilizado las formas tradicionales de organización social. Ahora la sociedad se piensa a partir de la metáfora de la red, es decir, como un conjunto de nodos interconectados que se vinculan a partir de la comunicación interactiva y asincrónica. El Estado se convierte en un nodo más - aunque importante- en las relaciones nodales de la sociedad de la información (Castells 2009, 44-6). Si bien en la época medieval existía un tipo de orden similar, la dinámica actual se centra en el intercambio de información, más que de mercancías.

\section{La memoria fragmentada: internet y la nueva construcción del recuerdo}

La era digital ha regresado a la humanidad a formas comunitarias y colaborativas de relacionamiento y construcción del conocimiento que se tenían antes del desarrollo y expansión del capitalismo. Por ejemplo, se puede citar el caso de Wikipedia, que es un espacio de creación de saberes en red y mediante la participación de los usuarios. Además, la dinámica gremial ha aparecido de nuevo a partir de las comunidades virtuales que se forman en foros y aplicaciones de interacción social.

Según Henry Jenkins $(2009,163)$, este nuevo movimiento social es producto del cruce de tres aspectos: a) la posibilidad de archivar, comentar, apropiarse y difundir productos mediáticos, b) el resurgimiento de la filosofía Do It Yourself de la subcultura punk en la creación de contenidos y c) la motivación de la participación de los públicos, a través de la circulación de información por parte de medios horizontalmente integrados.

Estos factores se enmarcan en el proceso de convergencia mediática que, como explica Jenkins $(2008,26)$, es producto de la transición de los medios analógicos a los digitales. Este cambio "altera la relación entre las tecnologías existentes, las industrias, los mercados, los géneros y el público", pues crea nuevas formas de producción y de consumo de contenidos. Para el autor, el intercambio de conocimiento mediado por la web es una práctica colectiva y recíproca (Jenkins 2009, 15), que se desarrolla dentro de un ambiente horizontal, ya que emisores y receptores participan en un mismo nivel. En el caso del relato histórico, las versiones oficiales desde el poder entran en discusión junto con las narraciones particulares de los públicos.

De acuerdo con Jelin $(2002,42)$, cuando se produce un cambio de escenario, se habilita una esfera pública nueva, donde se pueden incorporar las narraciones que han sido silenciadas y ocultadas por las estructuras de poder. En ese momento, se producen luchas por la representación del pasado entre los relatos oficiales y los subalternos en las que participan otros actores sociales.

La convergencia mediática y la globalización abren un escenario nuevo para la discusión sobre la historia: las aplicaciones de redes sociales, blogs, sitios web, podcast y foros 
de discusión. En esta gestión los blogueros, tuiteros o influencers vendrían a ser los nuevos emprendedores de la memoria, porque son los gestores de los relatos que circulan por las redes y, además, llaman la atención de los usuarios con sus productos mediáticos. Estos actores son los nuevos líderes de opinión.

Por otro lado, hay que considerar que el sentido de reconocimiento cambia en el mundo virtual, porque es el usuario quien legitima o no las versiones del pasado que se encuentran en la red. El prosumidor (acrónimo de productor y consumidor) construye su propio recuerdo a partir de su identificación con la información que busca en los espacios virtuales. Esta operación genera una desvinculación con la memoria colectiva estructurada desde el Estado y los grupos de poder, debido a la interacción deslocalizada de la web y a la formación de un nuevo sentido de comunidad que se basa en la interacción con individuos de diferentes contextos e ideologías. Este fenómeno también altera el sentido de pertenencia con lo local y con los relatos nacionalistas por la confrontación con la cultura globalizada.j

Según Melo (2011,91), el diálogo entre diferentes posiciones de los hechos pasados y las nuevas narrativas digitales fragmentan las versiones hegemónicas del pasado. El recuerdo - aunque mantiene su cualidad colectiva- deja de ser un constructo homogéneo; no representa a un solo grupo de pertenencia y se desterritorializa.

Por otro lado, de acuerdo con William Turkle, las evidencias digitales del pasado (como blogs, podcast, videos, imágenes o publicaciones en redes sociales) ocupan los estantes del archivo infinito, categoría que define a la web como "un archivo que está en constante cambio y tiene una eficacia infinita” (Turkle citado en Melo 2011, 95).

\section{El reto historiográfico: estudiar el pasado en la era del presente}

En el contexto actual, internet se ha convertido en el repositorio de la experiencia humana, ya que todas las acciones de los usuarios en las aplicaciones virtuales quedan registradas y guardadas eternamente. Por tanto, la memoria pasa a ser digital (84), lo que significa que se desprende de la materialidad de elementos simbólicos y afectivos para existir. Por ejemplo, los recuerdos familiares se conservan en álbumes de Facebook, lo que ha desplazado a la práctica de revelar fotos para guardarlas en portarretratos. En este escenario, el pasado se transforma en formato digital.

Actualmente, las innovaciones tecnológicas cambian las relaciones con el conocimiento. Como explica Michel Serres $(2013,21)$, las generaciones actuales utilizan Wikipedia para estudiar, lo que ha cambiado su manera de aprender, porque las herramientas virtuales "no estimulan ni las mismas zonas corticales que el uso del libro, de la tiza o del cuaderno". Agrega que los estudiantes ya no deben esforzarse por investigar, puesto que los buscadores hacen todo el trabajo: selección, análisis y presentación de datos (39). 
Como consecuencia, los jóvenes "no conocen ni integran, ni sintetizan" la información (21).

El cambio en el aprendizaje influye directamente tanto en el conocimiento sobre el pasado como en el ejercicio de la disciplina histórica. Por un lado, el uso del archivo digital modifica en la relación del investigador con la fuente física, que, de cierta manera, actuaba como lugar de memoria desde lo sensitivo. El olor, la textura, la observación de los detalles como la tipografía de un texto, entre otras percepciones, se pierden con la digitalización. Además, el uso de Skype para entrevistar a un testigo o la descripción de un hecho por medio del chat de una aplicación social no permite percibir detalles como el aumento de la respiración, la postura de las manos o los gestos que tienen las fuentes orales al recordar el pasado.

Frente a todo lo expuesto, ¿se podría considerar a la información digital como el nuevo lugar de la memoria? Según Melo $(2011,84)$, la red se está convirtiendo en un laboratorio para la disciplina histórica, debido a que almacena gran cantidad y diversidad de información. En función a esta necesidad, se desarrolló la Historia Digital. Según Orville Vernon Burton (citado en Melo 2011, 85), esta rama de la disciplina histórica es "el proceso por el que los historiadores son capaces de utilizar las computadoras para hacer historia en formas que sería imposible hacerlo sin ellas". Esta práctica se caracteriza por su relación con las herramientas tecnológicas como bases de datos, el hipertexto y las redes para construir y compartir conocimiento histórico (85).

Para Melo, la recuperación de la memoria no oficial en internet funciona mediante dinámicas similares a la de la historia oral, ya que toma como referencia fuentes alternas a las de los archivos nacionales tradicionales. En este punto, cabe mencionar la relación que se le ha dado a la dinámica social de internet con la de las culturas no occidentales, lo que permite comprender por qué en la era digital se están retomando las prácticas colectivas y cooperativas de construcción del conocimiento.

Sin embargo, los testimonios o evidencias del pasado difundidas y consumidas en internet presentan dos inconvenientes: el anonimato y la veracidad de la información. Estas fuentes deben atravesar por un proceso de curaduría que las orales, debido a que, como se dijo en líneas anteriores, muestran una versión subjetiva del pasado, en la que puede haber imprecisiones o datos falseados o inventados. Además, la web ofrece una realidad simulada, pues los usuarios construyen sus identidades, discursos y contenidos a partir de sus propias proyecciones.

La Historia Digital se ha preocupado por desarrollar metodologías de búsqueda de fuentes en internet, que van de la mano con técnicas de curaduría de información. Una de ellas es la minería de datos, que diseñó Turkle y consiste en "la extracción de conocimiento de grandes repositorios de información, estructurada o no en formato de texto" a partir del uso de la inteligencia artificial, las bases de datos y el análisis estadístico (Turkle citado en Melo 2011, 97). 
Cada vez crece el interés de los historiadores e investigadores por de acercarse a otros escenarios y ramas de conocimiento, para insertarse dentro de las dinámicas de la sociedad de la información. De esta manera, el relato histórico puede contribuir con la recuperación de esas memorias virtuales, que desaparecen rápidamente por el efecto mismo de la inmediatez de internet.

A manera de conclusión, el ritmo acelerado del tiempo en el que se desenvuelve la vida diaria, más la conexión constante con el mundo a través de la red cambiaron la relación de los individuos con el pasado. La necesidad constante de actualización convierte al recuerdo en una construcción efímera, que se pierde en la novedad.

En este escenario, las instituciones de poder y los colectivos sociales tienen que replantear sus estrategias de producción de memoria. Uno de los recursos más eficientes para este fin es la comunicación, debido al privilegio de la ubicuidad al estar distribuida entre los dispositivos móviles. El poder ya está tomando cartas en el asunto, pues cada vez se apropia de redes sociales como Facebook y Twitter para representar y legitimar su ideología y las narrativas oficiales que están de acuerdo con su discurso político. En Ecuador, este fue el caso de la explotación de la figura del expresidente Eloy Alfaro como efigie de la campaña propagandística y como lugar común dentro del discurso del expresidente Rafael Correa. En torno a la figura histórica de Alfaro, se elaboró una suerte de mitología por medio de relatos que engrandecieron su gestión política. Estas narraciones se emitieron continuamente en los medios públicos de comunicación y era un lugar común en los discursos del mandatario. El recuerdo de Alfaro aparecía cuando el gobierno de Correa buscaba legitimar sus decisiones o atacar a sus detractores.

En el ámbito personal, el colgar historias de vida en blogs, o subir los testimonios en YouTube se está convirtiendo en una tendencia de grupos sociales y comunidades, que han visto en la web 2.0 una plataforma para visibilizar sus recuerdos y dar a conocer su identidad. Por ejemplo, ahora la memoria sobre un evento familiar dura 24 horas como una "historia" en Facebook. Después de ese tiempo, se pierde en la nube y en el olvido de los usuarios. La fugacidad de este tipo de contenidos debilita el anclaje de los individuos con el sentido de memoria ya que se prioriza una necesidad de reconocimiento por un período de exposición mínima. Esta tendencia cuestiona sobre el sentido de trascendencia que se está consolidando en un tiempo en el que el legado desaparece en lo virtual.

\section{Conclusión}

La disciplina histórica tiene algunos temas pendientes. Existe en la mesa de análisis asuntos como el rol de las TIC en la formación del recuerdo en las nuevas generaciones; las dinámicas bajo las cuales se están construyendo las memorias colectivas dentro del ciberespacio y las ventajas y desventajas que implica ingresar a la red y las políticas que está 
utilizando el Estado para reivindicar su visión de la historia en tiempos de redes sociales. Si bien estos puntos fueron abordados muy brevemente en este escrito, podrían ser nuevos horizontes de investigación para la historia digital.

Aparte, es relevante que se analicen posibles metodologías de trabajo dentro de la web, ya que la información que yace en esta gran biblioteca puede servir para nutrir el proceso de recreación de los hechos. El reto, en este sentido, es desarrollar técnicas que faciliten al investigador comprobar la veracidad de las fuentes digitales, mediante el aprovechamiento de la cultura colaborativa de internet.

En suma, el pasado ha dejado de habitar en los pedestales de la conciencia colectiva contemporánea, debido a la hegemonía del presente. Toda expresión del ayer está siendo mirada desde la aceleración del tiempo, lo cual ha creado una problemática en cuanto a la recuperación de la memoria. Este ha sido uno de los "doble efectos" que ha tenido la tecnología comunicacional en la sociedad actual, ya que, si bien ha fracturado las visiones dominantes de la historia, y ha dejado salir a los relatos reprimidos por el poder, ha fraccionado al pasado y su sentido de verdad.

\section{Referencias}

Bustos, Guillermo. 2007. “La hispanización de la memoria pública en el cuarto centenario de fundación de Quito". En Etnicidad y poder en los países andinos, compilado por Christian Büschges, Guillermo Bustos y Olaf Kaltmeier, 112-34. Quito: UASB-E/Universität Bielefeld/CEN.

Bauman, Zygmunt. 2002. Modernidad líquida. Buenos Aires: FCE.

Castells, Manuel. 2009. “El poder en la sociedad en red”. En Comunicación y poder, de Manuel Castells, 33-81. Madrid: Alianza.

Halbwachs, Maurice. 1968. "Memoria colectiva y memoria histórica”. En La memoire colective, de Maurice Halbwachs, 209-19. París: Presses universitaires de France (PUF).

Jardim, José María. 2010. “Estado, información y archivos”. En Políticas y sistemas de archivos, de José María Jardim, 33-68. Ciudad de México: Secretaría General Iberoamericana.

Jelin, Elizabeth. 2002. "Las luchas políticas por la memoria”. En Los trabajos de la memoria, de Elizabeth Jelin, 39-62. Madrid: Siglo XXI.

Jenkins, Henry. 2008. Convergence culture: la cultura de la convergencia de los medios de comunicación. Barcelona: Paidós.

——_. 2009. Fans, blogueros y gamers: explorando la cultura participativa. Barcelona: Paidós.

Melo, Jairo Antonio. 2011. "Historia digital: la memoria en el archivo infinito". Historia Crítica

(43): 82-103. 
Moya, Laura y Margarita Olvera. 2010. “Conmemoraciones, historicidad y sociedad: Un panorama sociológico para la investigación”. En Independencia y Revolución: pasado, presente y futuro, coordinado por Gustavo Leyva, Brian Connaughton, Rodrigo Díaz, Néstor García Canclini y Carlos Illades, 437-60. Ciudad de México: FCE, Universidad Autónoma Metropolitana.

Serres, Michel. 2013. Pulgarcita. Buenos Aires: FCE. 